\title{
Les courtiers associatifs en développement dans le Sud-Est du Maroc
}

Entre politisation et dépolitisation

Mohammed Benidir

\section{OpenEdition \\ Journals}

Édition électronique

URL : http://journals.openedition.org/anthropodev/371

DOI : 10.4000/anthropodev.371

ISSN : 2553-1719

Éditeur

APAD - Association pour l'anthropologie du changement social et du développement

\section{Édition imprimée}

Date de publication : 1 septembre 2015

Pagination : 101-132

ISBN : 979-10-93476-02-5

ISSN : 2276-2019

\section{Référence électronique}

Mohammed Benidir, "Les courtiers associatifs en développement dans le Sud-Est du Maroc », Anthropologie \& développement [En ligne], 42-43 | 2015, mis en ligne le 01 décembre 2016, consulté le 20 avril 2019. URL : http://journals.openedition.org/anthropodev/371 ; DOI : 10.4000/ anthropodev.371

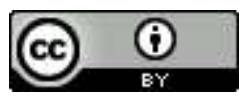

La revue Anthropologie \& développement est mise à disposition selon les termes de la Licence Creative Commons Attribution 4.0 International. 


\title{
Les courtiers associatifs en développement dans le Sud-Est du Maroc: entre politisation et dépolitisation
}

\author{
Mohammed Benidir
}

Cet article traite de l'un des chantiers de la fabrique des élites rurales, à savoir la reconversion des ressources tirées du courtage en développement dans le Sud-Est du Maroc. Suivant notre catégorisation empirique, la rétribution politique de l'activité courtière et les comportements stratégiques des courtiers prennent deux formes. Elles résident soit dans le franchissement soit dans le gommage des frontières entre courtage en développement et arène politique instituée. Dans le franchissement, nous avons affaire à des articulations entre la "politique des problèmes et des besoins " et la politique électorale, entre militantisme développementaliste et affiliation partisane, dès lors que les courtiers associatifs tentent de s'emparer du pouvoir décisionnel. La carrière du courtier s'encastre dans les parcours politiques et des trajectoires notabiliaires. Dans le gommage, certains courtiers entretiennent en revanche un intérêt déguisé vis-à-vis de la politique. Derrière la stratégie d'outsider, s'éclipsent des reconversions manquées, des prédispositions par rapport aux cadres de l'arène politique conventionnelle, des compromis et des arènes de compétition pour l'accès au rang de l'élite.

This paper deals with the rural elites' formation through the conversion of resources from the associative development brokerage in the Southeastern of Morocco. According to our empirical categorization, political rewards of broker activity and strategic behavior of brokers take two forms. They reside either in the crossing or in the scrub of the boundaries between development and brokerage politics instituted. Through this, we will be dealing with the links between the "political problems and needs" and electoral politics, and between developmentalist activism and party affiliation from the moment on when community brokers try to seize decision-making power. Career broker fits into the political path and trajectories of notables. In this context, some brokers maintain however disguised interest vis-à-vis politics. Behind this outsider strategy, missed con- 
versions, predispositions in relation to the frames of conventional politics, compromises and arenas of competition for access to the level of the elite are eclipsed.

\section{Introduction}

Dans le Sud-Est marocain, l'écrasante majorité des associations villageoises de développement apparaît " en dehors de l'arène directe de l'Etat après 1996 » (Zainabi et Ouhajou, 2005 : 8-9). Cette date coïncide avec les élections communales qui, suivies des élections législatives de 1997, ont permis "l'alternance consensuelle" au pouvoir et davantage d'ouverture de la part de l'Etat vis-à-vis des demandes socio-politiques émanant des organisations de la société civile. Les acteurs de la coopération au développement ont favorisé la naissance des " associations villageoises de développement " (AVD) dans le cadre d'une nouvelle configuration politique nationale. Les différents projets de développement, initiés en partenariat avec l'Etat et les organismes internationaux, et auxquels s'ajoutent ceux de $I^{\prime} I N D H^{1}$, conditionnent l'implication des acteurs de la " société civile ". Par conséquent, il se crée une intermédiation associative, voire un courtage en développement. II s'agit là d'une nouvelle carrière qui revendique des capacités techniques et communicationnelles, bref de nouvelles façons de faire (Cheynis, 2008 : 136) pour drainer différents projets vers les villages (les arènes) ${ }^{2}$. C'est à travers cette capacité de drainage que s'incarne l'intermédiaire entre destinataires de projets, dans les arènes locales, et institutions du développement.

D'après nos enquêtes, les trajectoires individuelles des courtiers associatifs du développement local puisent en général dans un éthos militant. Les devenirs courtiers s'appuient sur un arrière-plan de devenirs militants.

\footnotetext{
${ }^{1}$ L'Initiative nationale pour le développement humain (INDH) est un " chantier de règne " lancé par Mohammed VI en 2005. D'après la dernière plate-forme 2011-2015, elle se décline selon cinq programmes (le programme transversal, le programme de lutte contre la précarité, le programme de lutte contre l'exclusion sociale en milieu urbain, le programme de lutte contre la pauvreté en milieu rural et le programme de la mise à niveau territoriale).

2 Pour la définition de courtier en développement, voir (Blundo, 1995 : 74 ; Bierschenk, Chauveau et Olivier de Sardan, $2000: 7$ ).
} 
De leurs " points de vue ", l'intermédiation en développement est considérée comme le prolongement des actions militantes antérieures ${ }^{3}$. Cela démontre comment ces deux devenirs et trajectoires s'interpénètrent pour dépeindre les logiques d'entrée en courtage. Suivant notre catégorisation empirique, des profils peuvent être à cet égard repérés : une entrée par reclassement et recyclage du savoir-faire militant, l'effet de la coïncidence et du hasard dans le cadre des actions généralistes en développement, un appendice de la notabilité en place, l'entrée en courtage par la distanciation à l'égard de l'administration technique et, enfin, l'entrée par la formation et le renforcement des capacités. Une manière d'entrée en courtage correspond à une figure idéal-typique, voire à une arène. Respectivement, (anciens) militants, notables, agents sectoriels ${ }^{4}$ et généralistes font du courtage associatif en développement. Et celui-ci est une fabrique de nouvelles élites rurales, dans la mesure où il permet aux individus " excellents ", agissants et qui comptent de passer d'une arène à l'autre, tout en maintenant des liens entre les arènes imbriquées.

D'une manière d'entrée en courtage à l'autre, et d'une figure à l'autre, l'émergence des courtiers associatifs en développement dans les arènes locales du Sud-Est du Maroc engendre une reconversion des ressources et des positions de pouvoir. Les courtiers disposent de ressources, de compétences et de formes du capital, dans des arènes structurées par des enjeux et des logiques. En leur sein, les courtiers adoptent de subtiles stratégies pour tirer du profit, obtenir des crédits et remporter des "trophées" politiques. Ces stratégies signifient le maintien de leurs positions au prix $d^{\prime}$ 'une reconversion des espèces du capital en d'autres espèces, plus rentables et plus légitimes dans l'état considéré des instruments de reproduction, comme l'explique Bourdieu (1994: 8). Partant de là, le capital, qui consiste en la maîtrise des canaux techniques et communicationnels du

\footnotetext{
${ }^{3}$ Aux côtés de ces courtiers, les notables politiques traditionnels, qui sont en quête d'une professionnalisation, s'insèrent à leurs façons dans les opérations de courtage en développement. Leur but est d'éviter de laisser l'arène vide, disponible à une nouvelle élite rurale.

${ }^{4}$ Nous entendons par agents sectoriels les techniciens ou ingénieurs de l'Office régional de mise en valeur agricole de Ouarzazate ou de celui de Tafilalet (ORMVAO/T). II arrive fréquemment qu'ils découvrent la sphère associative du courtage en développement à travers leurs activités professionnelles.
} 
drainage des projets, se reconvertit en crédit. Le courtage en développement favorise l'émergence de nouvelles modalités de formation et de hiérarchisation des élites politiques qui ont progressivement transformé le politique et ses manifestations (Bourdarias, $2003: 24,34,35$ ).

Analyser les stratégies des courtiers associatifs dans les arènes structurées par des enjeux déterminés, laisse entrevoir la problématique d'enchevêtrement des logiques sociales et de superposition des dynamiques élitaires, tout en lui donnant un contenu empirique. L'enchevêtrement et la superposition ne s'opèrent pas entre modes de production délimités. À l'inverse, ils correspondent à des formes différenciées de drainage, d'accumulation et d'investissement des ressources de différents ordres, et emploient des canaux diversifiés dans des champs sociaux hétérogènes (Bako-Arifari et Le Meur, 2002 : 136).

Néanmoins, la reconversion/reproduction du capital en crédit et les superpositions des logiques sociales qui lui sont sous-jacentes impliquent une rationalité instrumentale. Celle-ci suggère que les courtiers associatifs en développement connaissent, avec des degrés différents, les arènes (mondes) au sein desquelles les ressources (les moyens) sont des maillons de causalité qu'ils manipulent pour atteindre les fins recherchées (crédits) les plus économiques possibles (Gellner, 1986 : 43). La malléabilité de leur capital, sa transformation et sa reconversion entrainent de multiples instrumentalisations et effets. L'imbrication des logiques fonde la rationalité instrumentale de l'activité courtière. Sur fond de cette imbrication (straddling) et de cette rationalité instrumentale nous souhaitons formuler I'hypothèse suivante ${ }^{5}$ : dans le Sud-Est du Maroc, les courtiers associatifs

\footnotetext{
${ }^{5}$ Cet article s'appuie sur les données de l'enquête de terrain récoltées dans le Sud-Est du Maroc en vue de la préparation d'une thèse de doctorat en cotutelle entre l'université Mohammed V Agdal-Rabat et l'université de Liège. Sur la base de l'analyse des trajectoires d'entrée en courtage en développement et les capacités acquises et mobilisées par les courtiers, nous décrypterons ici la position que ces derniers entretiennent au champ (politique) institutionnel ou les " qualifications qu'ils donnent eux-mêmes ", au sens où l'entend Bennani-Chraïbi (2011 : 56), aux actions courtières. Commencé en 2007, ce travail comprend un échantillon d'une vingtaine de personnes. Cherchant à appréhender leurs logiques et leurs " univers de sens » (Olivier de Sardan, $2008: 62$ ), nous avons eu recours aux entretiens semidirectifs. Répétés et vérifiés, tous les extraits d'entretiens ont été traduits de tamazight (langue berbère) et de l'arabe classique ou dialectal marocain vers le français par nos soins.
} 
en développement sont des entrepreneurs politiques (Lavigne Delville, 2000 : 165) qui franchissent ou gomment les frontières entre courtage en développement et arène politique instituée (notabilité). Dans le franchissement, nous démontrerons comment ils sont enclins pour de multiples raisons à transgresser ou à remettre en cause la différenciation (Lagroye, 2003 : 260-261), entre l'espace du socioculturel du courtage et l'arène politique instituée. Les frontières entre deux espaces, deux formes d'intermédiation se brouillent grâce à la convergence, nous le verrons en premier lieu, entre la compétition politique (politics) et les politiques publiques (policies), entre la "politique électorale " et la "politique des problèmes » (de Maillard, $2006: 40$ ) qui guettent l'exécution des projets de l'action publique par les acteurs politiques et institutionnels en place ainsi que celle " des besoins " publics de base auxquels sont confrontés les citoyens. Dans le gommage, certains courtiers entretiennent en revanche un intérêt déguisé vis-à-vis de la politique. Certes, la « distanciation » à l'égard de l'arène politique instituée et la neutralité/dépolitisation n'équivaudraient pas à "faire la dépolitisation ». Si cela implique une transformation active appliquée à un objet particulier, nous découvrons au gré des données récoltées que le mot " dépolitisation " évoque une passivité (Vedel, 1962 : 11). Autrement dit, si elle n'est pas expliquée par un certain nombre de stéréotypes actifs qui contrastent avec la politisation, s'agit-il là d'une autre façon de faire la politique ? Nous vérifierons en deuxième lieu comment la neutralisation et son corollaire qu'est la dépolitisation ne sont en contrepartie que d'autres manières de faire la politique et de pratiquer la participation.

Outre les entretiens, l'observation in situ nous a permis de construire une immersion et une empathie avec ces acteurs. Lesquelles nous ont aidé à écouter le discours spontané et la conscience pratique de ce qu'ils font à travers ce qu'ils sont et de ce qu'ils prétendent être, tout en neutralisant " axiologiquement » leurs " points de vue ». Je remercie Philippe Lavigne Delville, Sylvie Ayimpam, Amina El Messaoudi, Diego Sanges et Marie-Christine Polge pour leurs précieux commentaires et leurs remarques avisées sur une première version de ce texte. 


\section{La politisation du courtage et sa notabilisation}

Les rapports variés que les courtiers entretiennent avec l'arène politique instituée permettent d'appréhender les conversions des ressources, capitaux et compétences non politiques (militantes, expertes, gestionnaires, communicationnelles et techniques) dans la compétition politique (politics). Par là, ils fabriquent une stratégie qui trace des trajectoires notabiliaires. Toutefois, la relation qui se noue avec l'arène politique instituée se fait par différents modes quant à des "décisions, pratiques ou représentations qui tendent plus ou moins directement à définir les contours sans cesse renouvelés de l'espace politique » (Arnaud et Guinnet, 2005 : 17). Dans un premier temps, nous souhaitons étudier les raisons que les courtiers donnent au franchissement de ces contours. Dans un deuxième temps, nous démontrerons comment la conversion des ressources de différentes arènes contribue corrélativement à la conquête de la notabilité politique.

\section{Logique associative, stratégie politique et enjeu électoral}

Le premier mode de franchissement des frontières est l'enrôlement du courtier du développement dans l'arène électorale. Au cours des activités courtières, et en dépit de l'extériorité qui s'affiche a priori par rapport à ce qui lui semble politique - au sens conventionnel du terme -, le courtier soutient tel ou tel candidat durant la campagne électorale. Le cas d'un acteur associatif dans la chaîne montagneuse de Saghro en est une illustration de taille. Nous l'avons rencontré lors de la consultation électorale de 2007 et, bien qu'il déclare au préalable son apolitisme, il faisait alors campagne en faveur de l'actuel député PAM $^{6}$ de la circonscription électorale de Tinghir. En 2009, il se présente aux élections communales dans son douar d'origine. Au gré du processus d'enrôlement électoral, le courtier associatif en développement se mue mécaniquement en courtier électoral. II met sa communauté d'action et de préoccupations, créée lors de la mise

${ }^{6}$ Le Parti authenticité et modernité est un parti politique créé en 2008 par l'ami du Roi, Fouad Ali El Himma, autour d'un mouvement à dimension politique, le Mouvement pour tous les démocrates (MTD) qui a donné naissance à ce parti politique hors normes. Un groupe parlementaire a été créé avant même la fondation du parti. 
en œuvre des projets, au service d'un patron politique candidat à la députation. Dans ces conditions, la relation associationniste et contractuelle (Berdouzi, 1987 : 246) qui lie le courtier avec la population cible de projets se transforme en relation personnalisée et clientélaire. Dans le domaine associatif, celle-ci consiste en la requalification/reconversion, dans l'arène électorale, de la légitimité engrangée à travers l'arène de la mise en œuvre des projets de développement (Dahou, 2003: 156; Bennani-Chraïbi, 2004 : 42 ; Bennani-Chraïbi, $2011: 65)$. Tout en se nourrissant du capital associatif, le courtier associatif forge une notabilité sociale qu'il converti en légitimité (en crédit) politique (Berriane, 2009 : 188). En marge d'un atelier du démarrage d'un projet, ces conversions et requalifications politiques apparaissent dans les déclarations d'une conseillère à l'assemblée préfectorale d'Errachidia lorsqu'elle exhorte les courtiers associatifs à s'emparer des arènes politiques instituées :

"Prenez en vos mains les conseils (communaux). Tout passe par la politique. De la politique, on passe vers l'associatif et l'économique, etc. » (entretien, 2 septembre 2012, Agoudal (Imilchil)

Le chevauchement entre logique associative et logique communale est jusque-là un autre mode d'articulation entre notabilité et courtage (Benidir, 2011, 2014). Durant la campagne électorale des législatives, le courtier électoral cherche à se faire élire comme mandataire communal. En termes d'affiliation partisane, il faut distinguer deux voies. D'une part, la carte partisane peut s'identifier à celle du député qui est à la fois président du conseil communal ou municipal; et qui pratique une sorte d' " assimilation moléculaire des élites » (Geschière, 1996 : 86-87) 'à la marocaine', tout en oscillant entre clientélisme électoral et structures sociales tribales et ethniques. J'ai démontré ailleurs (Benidir, $2013: 345$ ) que le Janus notabiliaire député-président pratique cette stratégie de cooptation politique. Au moment des élections communales, il "intègre " les courtiers électoraux qui l'ont soutenu - lors des législatives - dans le parti auquel il est affilié. L'appartenance tribale et partisane des députés est, à quelques exceptions près, corrélée avec celle des présidents de communes. L'objectif consiste à donner au parti politique une dimension nationale, mais également à s'ériger en acteur influent vis-à-vis du parti, du gouvernement, des autorités locales, provinciales et centrales. Les élites assimilées ou cooptées dont font partie les courtiers associatifs et électoraux reconvertis en élus 
locaux - constituent une assise pour toute candidature du député aux instances représentatives au niveau local et national. Dès lors, l'ancrage local est une locomotive pour la consolidation de sa carrière politique, non seulement sur place, mais aussi dans un projet d'ascension politique à dimension nationale.

D'autre part, l'affiliation partisane des courtiers associatifs, qui ont assumé des postes de représentation politique locale, puise dans la socialisation politique antérieure. Bien qu'étant distancié (Ion, 1997), l'engagement de gauche corrèle avec la carte partisane choisie pour l'investiture à la notabilité communale. Empiriquement, des cas corroborent cette deuxième voie. Par exemple, le membre fondateur de l'association Amal Dades, connue pour le militantisme politique de gauche de ses membres, préside le conseil communal de Souk Lakhmiss lors des élections de 2009 au nom du PSU 7 . L'appartenance politique à ce parti de gauche remonte aux années de la formation universitaire à Agadir. Le président de l'association Oued El Hajaj, à Skoura, représente un cas identique. En créant l'association en 2002, dont le siège se trouve à Casablanca, il se fait élire lors des communales de 2003 ainsi que celles de 2009 dans son douar $d^{\prime}$ origine (la circonscription électorale $\mathrm{n}^{\circ} 32$ ) avec l'étiquette du $\mathrm{CNI}^{8}$. C'est avec la même étiquette partisane qu'il était candidat malheureux aux élections législatives de 2007. En parallèle, référons-nous au cas du président de l'association Tafergalte pour le développement à Agdez (Zagora) qui est en stradlling élu local de la commune rurale Mezguita. Au sein du conseil communal, il préside le Comité local du développement et des affaires sociales, culturelles et sportives. L'affiliation au PPS $^{9}$ va de pair avec sa socialisation politique et syndicale qui commence depuis le campus universitaire. Le dernier exemple est celui du président du conseil commu-

\footnotetext{
${ }^{7}$ Le Parti socialiste unifié fut créé en 2005 suite à une union entre le parti de la Gauche socialiste unifiée (lequel a rassemblé des partis et groupes d'extrême-gauche) et Fidélité à la démocratie, un parti qui est issu de l'Union socialiste des forces populaires en 2001.

${ }^{8}$ Le Congrès national Ittahadi (CNI) est le nouveau parti qui est né de la scission de I'USFP après le " gouvernement d'alternance consensuelle ".

${ }^{9}$ Héritier du Parti communiste du Maroc (Parti communiste marocain et Parti de la Libération et du socialisme), le Parti du progrès et du socialisme est légalisé en 1974, et il a tenu son premier congrès national en 1975.
} 
nal de Bouanan, au nom du PSU, à l'Est d'Errachidia. Pour lui, l'entrée en courtage du développement et dans l'arène politique communale est irriguée à la fois par ce qui reste du militantisme politique d'alors.

Du point de vue de certains acteurs, les opérations du courtage en développement ne sont pas en contradiction avec l'entrée en arène politique instituée, et qui se manifeste ici dans les tentatives de conquête du pouvoir décisionnel (Gaxie, 1978: 43). À Bouanane, en guise d'exemple, le cumul entre la figure d'élu et celle du courtier associatif s'explique par le fait que, si ce dernier "n'est pas en même temps élu local, il ne peut pas jouir d'un pouvoir décisionnel [solta taqrirya]» (entretien, Errachidia, le 12 janvier 2010). Certains d'entre eux considèrent que "les associations ont le même avenir que les communes " (entretien, El Kheng, le 16 janvier 2010). L'association Amal Dades représente un cas manifeste qui rend compte de la politisation progressive du courtage et de sa notabilisation. Comme vu précédemment, l'association abrite des militants à obédience socialiste (trotskiste et marxiste) et amazighe. Chaque année, elle organise le Festival Dades-Megoun en partenariat avec la Fondation Aknaray aux Pays-Bas et I'IDD $^{10}$, dont les thématiques vont de l'exclusion sociale à la marginalisation et l'élimination politique. En 2008, lui-même natif de Souk Lakhmiss, le responsable de la Fondation Aknaray critique les précédentes thématiques qu'il considère comme " ciblant une élite déterminée ". En droite ligne avec la politisation lagroyenne (Lagroye, 2003 : 260-261 ; AïtAoudia, Bennani-Chraïbi, Contamin, 2010), les courtiers associatifs transgressent et remettent en cause la différenciation entre l'espace du courtage - socio-culturel pour l'essentiel - et la notabilité politique. Les propos du responsable de la fondation cité ici sont significatifs :

"Vu la réputation du festival (Dades-Megoun), il doit être intéressé, en premier chef, aux problèmes de la zone sur tous les plans... En particulier, la priorité doit être accordée à la démocratie locale, à la participation dans la vie politique. [...] Les élections locales jouent

\footnotetext{
${ }^{10}$ Le réseau Immigration, Développement, Démocratie (IDD) fut officiellement fondé en 1999. Il a été créé, suite à un débat sur le développement au sein d'un réseau de la gauche marocaine en France, à I'ATMF (Association des travailleurs maghrébins de France) à partir de 1989, et en Europe, au sein du CADIME (Coordination des associations marocaines démocratiques en Europe), à partir de 1993.
} 
un rôle important dans l'acquisition de plusieurs droits. II ne faut pas toujours compter sur le changement d'en haut tout en oubliant le rôle des partis politiques et les associations. Là, il faut aider les jeunes afin qu'ils soient intégrés dans la vie politique. " (Abdellah Talal, 2008) ${ }^{11}$

Ce dernier cas démontre comment la qualification/reconversion des ressources injectées dans l'interface développementaliste permet aux courtiers-militants de réaliser un ancrage dans l'arène politique instituée, tout en politisant les discours, les enjeux et les représentations après des années de dé-politisation et de contestation. Aux élections communales de 2009, ils évincent d'anciennes élites politiques qui avaient siégé pendant trente ans au conseil communal de Souk Lakhmis. La transition de la logique de contestation vers celle de l'intégration à l'enceinte communale, et celle du discours misérabiliste de la lutte pour la dé-marginalisation vers celle de la participation au développement et à la politique sont autant $d^{\prime}$ 'indices qui confirment l'hypothèse de constitution de nouveaux notables et la fabrique de nouvelles élites rurales. Cependant, celles-ci sont confrontées, y compris au terme de cet exemple, à un double-bind, à une injonction contradictoire : comment éviter de ne pas renoncer à la voie militante héritée et même à ses paramètres distanciés tout en prenant position dans l'arène politique instituée ? Le maintien des réseaux de l'activité courtière en développement et parfois même la construction d'une distance envers les courtiers intégrés à la commune permettent de gérer ce paradoxe. Les courtiers qui ont préféré rester en marge de l'arène communale gardent le statut du relais associatif et de l'activiste socioculturel', ce qui produit de nouvelles recompositions sociales. Le mariage de raison et les alliances entre camarades d'antan, courtiers associatifs et élus locaux d'aujourd'hui ont finalement débouché sur des chaînes de conflictualité et d'opposition; notamment sur les choix, les prises de position et

${ }^{11}$ Cet entretien avec Abdellah Talal, président de la Fondation Aknaray, est publié dans Azqour $n^{\circ} 4-5,2008$, p.2, une publication dite " intellectuelle, culturelle et journalistique complète " éditée par l'association Amal Dades (AAD) en collaboration avec l'association IDD, I'ATMF et la fondation Aknaray. D'où le choix de la thématique du dernier festival portant sur "la gestion des affaires locales: quels enjeux et quelles approches » en marge duquel la thématique de "l'État régional dans le Maroc démocratique » a été abordée. 
les décisions prises dans le cadre de l'implémentation des politiques publiques locales.

Un des effets induits de l'articulation du courtage en développement à l'arène politique instituée est la convergence entre politics et policies (Catusse et Karam, 2009 : 98). Selon des cas typiques, il s'avère que les courtiers sont captivés par les arènes (Faure, 1997: 77) de la mise en œuvre des politiques publiques lorsqu'ils interprètent à terme ses référentiels, mais aussi par celles relevant de la compétition politique entre acteurs concurrents. Nous nous arrêterons sur deux exemples. Le premier relève du président de la commune rurale Souk Lakhmiss qui n'hésite pas à critiquer les projets et les programmes de l'Etat. En termes de gestion communale, il insiste sur la malsaine concertation qui s'opère au sein du comité local du développement humain. Ce dernier étant l'instrument de planification stratégique du développement local et de gouvernance territoriale dont le but est d'articuler les dynamiques locales des associations avec les initiatives locales des élus. Selon lui :

"[...] la concertation reste un vœux pieux. Par exemple, l'élaboration du PCD (plan communal du développement) est confiée techniquement à un bureau d'études, alors que les élus n'ont aucune marge de proposition. Ils n'ont qu'à l'autoriser pour ne pas freiner les projets de l'Etat qui sont mal diagnostiqués par des techniques loin de la population à la base et ses besoins réels " (entretien, Kelaat Megouna, le 8 février 2010).

Ces propos révèlent que, dans le sens où la controverse se concentre ici plus sur le référentiel de la politique publique que sur la compétition politique, la concertation et la participation se plient aux procédures/techniques des projets mis en place (Bono, 2009: 244) dans une arène où le politics est vidé de toute sa substance.

L'entrée des courtiers dans la politique conventionnelle s'inscrit parfois dans un contexte où prédomine le primat de la compétition politique, voire même celui de la surpolitisation des arènes. Cette surpolitisation réside dans le fait que les coalitions associatives vont de pair avec les interférences politico-partisanes. A Bouanane, les élus de l'opposition, qui sont 
issus en majorité de I'Istiqlal $^{12}$, parrainent une organisation fédérative qui leur est propre (Forum de solidarité pour le Sud-Est). À l'inverse, la majorité est alliée avec les élus du PAM et est présidée par un membre du PSU. Ceux-ci sont organisés dans le cadre du réseau associatif appelé : "Union des initiatives du développement de Bouanane ». Les deux associations sont membres du CLDH. D’après le président :

"[...] l'opposition de l'opposition est parfois incompréhensible. Si un tel projet touche la population toute entière et s'implante dans la zone de leur élection, ils n'ont qu'à participer et valider le projet. Mais s'il n'était pas le cas, ils votent non, bien qu'ils constituent une minorité au sein du conseil. " (entretien, Errachidia, le 12 janvier 2010)13

Une situation qui exacerbe les conflits entre les membres du conseil communal et ceux du comité local du développement humain, tout en engendrant diverses formes d'instrumentalisation et de patronage.

\section{Entre le «douar », la « rue » et le « couloir»}

Que sont alors devenus les courtiers associatifs ? Au gré des exemples cités, la conversion des "capitaux " techniques accumulés en " crédits " politiques et la transfiguration du courtage en notabilité s'avèrent insuffisantes si elles ne s'accompagnent pas de la qualification des capacités politiques, et qui réfère à une nouvelle manière de faire le militantisme. Le cas des leaders de la Fédération des associations du développement de Tinghir (FADT) nous offre un exemple saillant de la politisation du courtage et de sa notabilisation progressive. Ces leaders s'emparent des savoir-faire

\footnotetext{
12 Formant la composante du mouvement national, le parti de l'Istiqlal (Indépendance) est créé en 1944. Bien qu'il semble être le parti "clanique " de certaines familles d'Ahl Fès, il remporte des sièges même dans les zones montagneuses, désertiques et semi-désertiques les plus enclavées.

${ }^{13}$ En parallèle, un membre du Réseau des associations du développement du Grand Ferkla qui est également un élu communal au nom du PJD explique l'ambiguïté de cette opposition par le fait que celle-ci "croie que la majorité est dominante et d'exclusion, alors que la seconde critique la première comme s'il s'agit d'un obstacle qui dérange » (entretien, Tinjdad, le 22 février 2014).
} 
techniques et de la maîtrise des contacts stratégiques avec les bailleurs de fonds, non pas seulement pour endosser la figure du courtier dans la mise en œuvre des projets, mais aussi pour prétendre à des responsabilités représentatives. Bref, ils font du développement pour la politique. L'ethnographie de leur " militantisme développementaliste ${ }^{14}$ confirme la possibilité de requalifier et de reconvertir des savoir-faire d'une arène à l'autre. En l'occurrence celle du courtage et du militantisme vers celle de la compétition politique qui se traduit par (les tentatives de) la conquête de notabilité communale et parlementaire.

En s'appuyant sur ce que certains appellent « la foi développementaliste ", notamment dans son espèce contestataire et participationniste (Bierschenk, Chauveau et Olivier de Sardan, 2000 : 24), I'initiation des stratégies politiques se base sur les éléments suivants: la mobilisation contre la mauvaise gestion des affaires locales par les élites politiques en place ; la rédaction des lettres de dénonciation ou des doléances adressées aux instances gouvernementales, représentatives et partisanes; et les prises de position par rapport à des décisions publiques au niveau local. En effet, la FADT avait organisé des manifestations contre l'enclavement de la région. Affiliés en plus au PJD, les leaders de la FADT construisent un discours d'autorité sur la nature des problèmes à résoudre et catalysent par là même des formes de protestation latentes et balbutiantes. Ils cristallisent un ancrage local (Catusse et Karam, 2009 : 100-104) pour s'emparer d'une légitimité de la représentation politique ultérieure, à savoir celle de la notabilité communale et, plus tard, parlementaire à l'heure des élections législatives anticipées de 2011. Là, ils se plient malgré eux aux règles du jeu partisan local, ce qui sape pour une part l'image de porte-parole de la protestation à laquelle les courtiers associatifs affiliés au PJD pourraient prétendre (Catusse et Zaki, $2009: 85$ ).

La notabilisation des courtiers associatifs, à travers le cas de la FADT récapitulé ci-dessus, est façonnée par la " conversion circulaire " des ressources et des savoir-faire (Bennani-Chraïbi, 2011 : 71) et l'« ubiquité réticulaire " (Blundo, 1998: 38) dans différentes arènes et réseaux. Ces derniers sont ceux du "douar ", de la " rue " et du " couloir » au sein des-

${ }^{14}$ Pour l'évolution de leur mobilisation, cf Benidir (2012 : 33-37). 
quels divers modes de légitimation s'irriguent mutuellement. La représentation électorale, le discours idéologique, la compétence technique, la maîtrise des contacts stratégiques - tant avec les bailleurs de fonds qu'avec les associations des ressortissants - et, enfin, la construction d'un réseau relationnel constituent corrélativement autant de modes légitimant l'entrée des courtiers dans l'arène politique instituée. Le courtage en développement qu'exemplifie jusqu'alors la FADT, autant pour des raisons idéologiques (légitimité du capital associatif et primauté du local dans le discours) que pour des raisons techniques (compétences et réseaux de relations) (Boucher et Villalba, $1990: 41$; Catusse et Zaki, 2009 : 90), détermine la conquête de la notabilité communale et même parlementaire. En ce sens, ce sont les associations qui, à travers les capacités dont les courtiers sont porteurs, influencent en dernier abord les processus de mobilisation-participation autour du pouvoir municipal ${ }^{15}$.

La politisation du courtage et sa notabilisation estompent-elles la spécificité du courtier ou des courtiers telle que nous l'avons décrite à plusieurs reprises ? Cette question centrale qui a été soulevée par Giorgio Blundo à propos du Sénégal est d'actualité en ce qui concerne le Sud-Est du Maroc. A l'aide d'une ethnographie en longitudinal des courtiers, la multiappartenance à des arènes multiples et imbriquées et la conversion circulaire des ressources, des arguments et des savoir-faire démontrent comment le courtage constitue un tremplin à une carrière politique et/ou à une ascension politique (Blundo, 2000 : 73-83). En entrant dans l'arène politique instituée (enrôlement électoral, conseil communal, parlement, prises de position par rapport à la gestion des affaires publiques locales, affrontement avec les autorités locales, implémentation des politiques publiques), les courtiers passent de l'insider culturel à l'outsider politique (Blundo, 2000 : 104 ; Benidir, 2010 : 104), étant donné qu'ils sont en quête d'une nouvelle légitimité. Ils cherchent, dans une requalification des opérations du courtage quant à ses actions et ses ressources techniques - ou celles politiques qui les précèdent synchroniquement ou diachronique-

15 Inversement à R. Balme qui soutient l'idée selon laquelle « la dynamique de participation à l'origine du renouveau associatif, souvent attribuée aux nouvelles catégories sociales intermédiaires, semble ainsi s'estomper pour laisser aux municipalités la maîtrise relative des processus de mobilisation des associations autour du pouvoir communal " (Balme, 1987: 601). 
ment -, à s'imposer face aux élites en place pour influer sur les décisions prises ou à prendre, mais aussi à conquérir le pouvoir dès lors qu'ils les évincent de l'arène. Cela confirme davantage l'hypothèse d'émergence de nouveaux entrants et de la fabrique de nouvelles élites sur fond de la conversion politique des ressources du courtage. Une telle émergence par évincement, abstraction faite des alliances qu'elles peuvent contracter dans certains cas, bouleverse les rapports de force en faveur de la gestion des affaires publiques selon un modèle associatif, ce qui met en branle les intérêts des élites en place.

\section{Stratégie d'outsider ou comment faire la politique autrement}

La stratégie d'ascension politique prend ici une image qui rivalise avec la précédente. Une autre catégorie de courtiers défend une vision neutralisante et dépolitisée du courtage en développement. Si les courtiers, dans leur stratégie de notabilisation, sont qualifiés d'entrepreneurs de politisation, quid de ceux qui ont une vision neutralisée ou dépolitisée dans la mise à jour des pratiques associatives du courtage? Parle-t-on d'entrepreneurs politiques de dépolitisation ? Au cours des activités courtières, ils visent à neutraliser un certain nombre d'activités ou thématiques, notamment lorsqu'ils désactivent ou dissimulent toute connotation supposée politique, y compris la compétition électorale. Ils entretiennent un désintérêt ou à juste titre un intérêt déguisé à l'égard des affaires publiques villageoises, notamment en ce qui concerne les anciens cadres et critères (Mendras, 1962 : 261). De "leur point de vue ", les associations, actrices à part entière du courtage entre populations et responsables de tout bord de projets, sont conçues comme étant des structures apolitiques ou même "purifiées » des bassesses de l'action politique (Al-Sayyid Said, 2004 : 76). Cela donne l'impression que le courtage en développement ne peut se faire que sur la base d'une dépolitisation des enjeux et d'une recherche de consensus, à partir de la valorisation du "petit", du " concret " et du " ponctuel » (Bennani-Chraïbi, 2003 : 352). Ces attitudes, ces logiques, qui accompagnent les opérations de courtage, portent en ellesmêmes une vision modificative des relations qui se nouent et se dénouent entre État, collectivités locales, autorités locales et sociétés locales, arènes auxquelles s'adressent les projets. Il s'agit là aussi d'une vision fonctionnaliste qui technicise ce rapport et le dépolitise davantage (Abelès, 1986 : 
257), sachant que les enjeux de la rétribution réelle de telles opérations se trouvent en dehors de l'ordre politique local. Dans tous les cas, nous verrons que la neutralisation, la dépolitisation et encore la technicisation des activités courtières participent en réalité à la stratégie de l'outsider (Catusse et Karam, 2009 : 97). Elles forgent par là même de nouvelles manières de faire la politique ainsi que celles de (re)penser la participation associative.

En droite ligne avec notre catégorisation empirique des trajectoires d'entrée en courtage, deux figures idéal-typiques illustrent différemment des logiques représentationnelles et stratégiques (Olivier de Sardan, 1995 : 126-127) de la neutralité et de la dépolitisation. La première a trait aux généralistes dont les actions courtières en développement s'appuient sur un savoir-faire transversal qui désormais ne recourt pas aux mêmes registres d'intelligibilité (Nay et Smith, 2002 : 14). La deuxième relève des agents sectoriels qui sont entrés en courtage par ce que l'on a qualifié de "l'autre manière d'aller au peuple ", tout en s'éloignant de la pure technique et se distanciant, pour une grande part, de l'administration. Ils font, dans l'interface associative, ce qu'ils n'ont pas pu faire dans l'administration sectorielle où ils sont attachés.

\section{Savoir-faire transversal et neutralité}

Les entretiens ethnographiques menés avec les généralistes révèlent la différenciation des formes de penser et de faire la dépolitisation/neutralisation des opérations du courtage. Le premier cas est celui d'un courtier généraliste dont la stratégie consiste en la quête, fut-elle avortée, d'une néo-légitimité notabiliaire dans laquelle la neutralité dissimule une manière de faire la politique autrement (Catusse, 2002 : 308309 ; Bourqia, 2011 : 101 et 113). Avant que son enrôlement électoral dans les législatives et sa candidature aux communales qui les suivent débutent, il nous déclare: "même si le développement est par essence politique, je ne prétends pas, à travers l'association et les projets, faire la politique " (entretien, Saghro, le 10 novembre 2006). Comme nous l'avons mis en exergue du point de vue théorique, il fait en apparence référence à un gommage déguisé des frontières du politique. Néanmoins, le comportement d'apolitisme déclaré recèle les tentatives de s'emparer d'une nouvelle notabilité par le courtage caractérisé par des activités diversifiées et 
généralistes. À l'instar de son premier démarrage en courtage qui n'a pas été abouti comme nous l'avons mis en lumière ci-avant, son entrée en arène politique instituée a échoué. II a été battu aux élections communales. Bien qu'étant évincé de l'association villageoise par son concurrent vainqueur et membre de l'association, ses actions courtières en développement se maintiennent au niveau supra-local. II joue la fonction du courtier au nom de la fédération ${ }^{16}$ et non plus au nom de l'association dont il fut fondateur :

"J'interviens seulement à l'échelle communale à travers la fédération des associations et non pas dans le cadre de l'association villageoise qui se trouve dans le village natal » (entretien, Ouarzazate, le 08 février 2011) ${ }^{17}$.

Cela signifie que l'échec d'entrée en arène politique instituée contraint le courtier à un changement d'échelle d'intervention.

Concernant le deuxième cas, les acteurs généralistes en développement confondent avec succès la neutralisation vis-à-vis de la sphère conventionnelle de l'arène politique et la participation pragmatique à celle-ci. Le cas de l'association Akhiam dans le Haut Atlas oriental en est une illustration exemplaire. Les propos de son président expliquent métaphoriquement comment les postures généralistes, pragmatiques et neutralisantes font une entreprise commune :

"Dans chaque cheval qui vient, il faut y monter. S'il mène vers le développement on y reste, sinon on y descend " (entretien, Imilchil, le 26 septembre 2009).

${ }^{16}$ Comme en témoigne sa fonction de relais dans le cadre du projet " Conservation de la Biodiversité dans le Saghro ». Le projet est financé par le Programme des micro-financements du Fonds de l'environnement mondial (PMF/FEM- PNUD), voir Alifriqui (2012:5).

${ }^{17}$ En quittant en fin de compte la fédération, il porte son savoir-faire en matière de courtage en développement en dehors de son village. Actuellement, il est membre de plusieurs associations à Ouarzazate centre (association Oasis de la femme et de l'enfant pour le développement et la solidarité, Association des populations de montagne (APM)-succursale de Ouarzazate, association de quartier El Ouahda et Association des apicultures de Zagora), entretien, 31 décembre 2012, Ouarzazate. 
L'association fait partie d'un univers multi-varié des projets. En l'occurrence, elle est membre du Comité provincial du développement humain $(C P D H)$, dans la mesure où il représente la zone montagneuse de la province d'Errachidia. Selon le président, elle n'a pas une force de proposition, mais cela ne l'empêche pas de participer aux débats qui se tiennent au sein de ce comité. Quant au conseil communal de Bouzmou où se trouve l'association, celle-ci est membre du Comité local du développement humain (CLDH). Selon ses dires, la position du courtier associatif visà-vis de l'arène communale varie entre la neutralité et la participation pragmatique, eu égard à l'intégration de la dimension collective, tribale et notabiliaire dans le montage et la mise en œuvre des projets. Dans le montage des projets, il a recours à ce qu'il considère une " mobilisation villageoise ». Tel est le cas du projet relatif à « la lutte contre l'érosion des sols dans la plaine alluviale dans la vallée d'Imilchil et (pour) limiter les impacts sur l'agriculture ", un projet qui a été financé par Hydraulique sans frontière-Méditerranée (HSF). Au sujet des relations avec les communes, le président de l'association déclare:

"[...] les services de la commune et ceux de l'Office de mise en valeur agricole ${ }^{18}$ au niveau local doivent accompagner le comité du pilotage dans le cadre d'une approche participative. [...] La commune rurale a besoin de nos capacités techniques. Pour nous, nous en avons besoin en termes de son apport institutionnel que conditionnent les projets. » (entretien, Imilchil, le 26 septembre 2009)

Pour sa part, la position du président du conseil communal de Bouzmou, dans la vallée d'Assif Mellouln (Imilchil), s'inscrit dans le même ordre d'idée. En marge d'un atelier du démarrage de projet sur le " Renforcement des capacités des femmes du Haut Atlas oriental, pour leur autonomisation économique et sociale " dont I'association Akhiam est l'acteur porteur, il déclare à ce sujet :

\footnotetext{
${ }^{18}$ Organisme public doté de la personnalité morale et de l'autonomie financière placé sous la tutelle du ministère de l'Agriculture et de la Pêche maritime. Il est implanté à Ouarzazate et à Errachidia depuis le début des années 1960. Outre sa vocation essentiellement agricole, l'Office a comme objectif de créer un dynamisme et de disséminer le développement dans sa zone d'action. Généralement, les limites de la zone d'action de l'ORMVA d'Ouarzazate ou celui de Tafilalet ne correspondent pas à celles de la province administrative.
} 
"L'association Akhiam est parmi les associations très dynamiques. Elle est membre du comité local du développement humain. Je suis parmi les gens qui la soutiennent. N'est-ce pas un seul grain de blé ne fait pas à lui seul du pain (urda itgga yan ird aghroum). " (entretien, le 2 septembre 2012, à Imilchil (Agoudal)

La neutralisation qui s'appuie sur la posture à la fois généraliste et pragmatique signifie-t-elle une instrumentalisation réciproque entre l'association Akhiam et la commune rurale de Bouzmou ? S'agit-il là d'un alibi politique ? Bien entendu. Les propos cités supra informent sur le parti pris d'instrumentalisations mutuelles ou bien ce que Lagroye appelle les " cannibalisations réciproques » (Lagroye, 2003 : 371) entre l'espace social du courtage en développement et l'arène politique conventionnelle ; lesquelles cannibalisations mettent les gens du développement et les gens du pouvoir en coexistence pacifique (Bierschenk et al., 2000 : 32). Par ailleurs, la neutralité s'efforce d'être volens nolens un alibi politique, dans le sens où elle s'instrumentalise à l'égard d'une catégorie de bailleurs de fonds ${ }^{19}$. Dans la valeur que ces derniers exigent pour le financement de certains projets, les courtiers de l'association Akhiam déclarent que celle-ci n'est ni politique, ni religieuse, ni humaniste, mais bien neutre. La neutralité signifie ici :

"[...] une sorte de morale politique définie à partir d'un système de valeurs plus qu'une catégorie de la science politique qui pourrait se définir à partir de faits constatés sans jugement de valeur présupposé » (Vedel, $1962: 12)$.

II s'agit d'un système de valeurs, mais aussi d'un alibi qui répond aux exigences et aux tendances des bailleurs de fonds et des pouvoirs publics. "La politique de bons offices ", installée entre l'association Akhiam et les acteurs publics et institutionnels, s'explique aussi par l'absence d'un discours militant et misérabiliste de déni ; un discours dont on peut rendre compte ailleurs, en l'occurrence dans les zones de Dra, Ghris, Tinghir et

19 Tels que I'ONG Solidarité Echange Nord Sud (SENS), I'Agence Japonaise de Coopération Internationale (JICA), I'ONU Femmes, INDH, ORMVAT, I'Association Marocaine de Solidarité et de Développement (AMSED), le PNUD, I'UE, I'Agence Micro Projets, Hydraulique Sans frontières (HSF), le Service de Coopération et d'Action Culturelle (SCAC) de l'Ambassade de France, le Comité Catholique contre la Faim et pour le Développement (CCFD), etc.. 
Dades. Pourtant, la zone d'Imilchil est l'une des régions les plus reculées et enclavées en termes de développement humain au Maroc.

Parmi les exemples les plus probants, on peut également citer celui de l'association Oasis Ferkla pour l'environnement et le patrimoine (OFEP), à Tinjdad, dans la province d'Errachdia. À l'instar de précédents cas, le caractère généraliste des activités courtières est bel et bien mis en exergue. II est même cité dans le statut de l'association : "son action ne se limite pas à un domaine spécifique " (entretien avec deux membres au siège de l'association, Tinjdad, le 9 janvier 2010). À première vue, il s'est avéré d'une part que les comportements stratégiques relatifs à la neutralisation et à la dépolitisation concernent les hommes ${ }^{20}$ et non pas les institutions administratives et représentatives. Nous rappelons qu'au début de l'entretien, l'un d'entre eux déclarait que " ces gens (de l'association rivale et de la commune), nous ne les connaissons pas" (entretien avec un membre de l'association, Tinjdad, le 9 janvier 2010). La rivalité associative impose au courtier en développement d'adopter une position d'outsider. D'autre part, si l'on considère l'échec du président de l'association dans les élections parlementaires qui coïncide avec le début de son entrée en courtage en développement, force est de constater que la dépolitisation et la neutralisation représentent toutes deux un apolitisme tactique. Comment en arrivons-nous à une telle situation ? En quête d'une nouvelle légitimité dans l'arène locale de Tinjdad, dans laquelle règne de facto des logiques ethniques conflictuelles, le courtier en développement s'appuie sur une micro-stratégie de requalification par la dépolitisation dans une perspective probable de repolitisation. La neutralité est un gommage relatif des frontières de la politique. Ressource de légitimité, elle est destinée à mieux s'imposer face aux courtiers belligérants, et qui sont pour une part des élus communaux en straddling. En tant que stratège dans l'arène locale, ces tactiques définissent les circonstances du redéploiement dans les arènes vis-à-vis des groupes au titre et pour le compte desquels ils se dévouent dans le cadre de la mise en place des projets "integrés ". Se distinguer de la politique (Catusse, 2002: 309), tout en mélangeant

20 Inversement à ce que peut signifier la politisation qui renvoie aux institutions (Vedel, $1962: 12)$. 
l'interpersonnel et l'institutionnel, est une règle pragmatique (Bailey, 1971: 231) de disqualification des courtiers concurrents qui, relevant de la capacité et du stratagème, visent à se repositionner et à prendre parti dans l'arène locale. En bref, il s'agit d'une stratégie où vont subrepticement de pair les intérêts politiques, et même " un intérêt pour une certaine tendance politique " (Merle, 1962: 44) ainsi que les activités courtières en développement.

\section{Une autre manière d'aller au " peuple » et de faire la politique}

Concernant les agents sectoriels, y compris les ingénieurs et les techniciens, l'inverse du parti pris souligné par Paul Pascon est d'importance à plus d'un titre, afin de décrypter une autre manière de faire la politique par le courtage en développement. Sur ce point, il déclare que ceux qui "vont au peuple [...] ne trouvent pas d'emblée des interlocuteurs, lesquels puissent les aider à dépasser le dilemme productivité/profit " (Pascon, 1980 : 36). Or, le retour au " peuple » à travers l'intermédiation associative nécessite le retrait de la pure technique liée aux projets intégrés de l'administration sectorielle à laquelle ils sont bureaucratiquement attachés. Dans ces conditions, la logique populaire des interlocuteurs l'emporte sur celles technicienne, bureaucratique, administrative et de classe (Pascon, ibid.). Au lieu de se limiter au rôle d'agent et d'exécuteur de I'action sociale et politique décidée par l'Etat et son administration sectorielle, ils instrumentalisent à leurs façons les projets techniques et bien d'autres. Ici, la connaissance des "bricolages" et les "bavures", pour reprendre les formules de Pascon, ou les dérives dans le sens où l'entend Olivier de Sardan, renforcent les tendances de ce retour au peuple et la distanciation à l'égard de la pure technique.

Commençons par l'agent sectoriel qui a cofondé l'Association du développement dans la vallée du Dra (ADEDRA). L'intérêt de dépasser le dilemme productivité/profit le conduit à repenser la logique populaire des interlocuteurs et des intermédiaires en développement. Il avoue :

"L'ADEDRA a pratiqué entre 1996 et 1997 une approche descendante caractérisée par la prédominance de la vision des leaders, membres du bureau de l'association, qui se sont substitués à la population. En raison du manque d'expérience, mais aussi sous la pression de réalisation de ses premiers projets, elle a entrepris des 
opérations à la demande des bailleurs de fonds afin de gagner de la crédibilité auprès du groupe cible. L'association s'était rapidement engagée avec des personnes physiques étrangères ayant des objectifs mal-définis ou tout simplement latents. La participation populaire nécessite la recherche d'une façon de faire facilitant l'intrusion et l'acceptation par la société locale à travers l'action sociale." (Zainabi, 2003 : 55)

Suivant les dires de cet agent sectoriel, la conception qu'il donne aux actions de l'association nie toute intermédiation associative individuelle et courtage en développement. Comme le révèle le rapport d'activités de l'association, celle-ci :

"[...] entend promouvoir des méthodes assurant et facilitant la participation de tous et surtout réduire le rôle d'intermédiaires par le renforcement des capacités locales » (ADEDRA, 2005 : 14).

Son étude d'expertise dans le cadre du "World Development Report 2003: Sustainable Development in a Dynamic World" est de la même veine. Il affirme qu'il convient de se prémunir contre la reproduction, par quelques associations, $d$ 'un certain modèle bureaucratique et administratif et, surtout, contre la fonction d'intermédiation développée par certaines associations sous prétexte de la professionnalisation du travail associatif. Parallèlement, le rapport d'activités de l'association insiste sur l'idée selon laquelle est :

"[...]plus grave encore cette prolifération d'intermédiaires qui témoigne du développement d'un système de captage pour ne pas dire de détournement de l'aide extérieure destinée à l'origine aux groupements de base " (ibid.).

Cependant, cette manière autre de penser la participation populaire, qui est - semble-t-il - en déphasage avec le courtage, est parsemée d'embûches. Fortement soutenue par la coopération technique allemande (GTZ), I'ADEDRA se heurte actuellement aux problèmes de financement. Les actions dites d'auto-promotion ont disparu dans la vallée, dès lors que l'appui de la GTZ a pris fin. Le cadre détaché de l'agriculture, étant l'agent sectoriel qui s'est reconverti en courtier associatif en développement, était le directeur du PROLUDRA. II a cédé la présidence au big men de Zagora 
(président de la municipalité de Zagora et ex-député du Mouvement populaire $\left.{ }^{21}\right)$. L'association se limite seulement aux ressources glanées de l'INDH, et qui sont modestes par rapport à celles de la GTZ. En réalisant par là même une ascension socioprofessionnelle, le courtier devient expert. II est muté à Rabat pour rejoindre l'unité de gestion du programme d'appui aux actions de réparation communautaire en faveur des régions touchées par la répression et les violations des droits de l'homme. Vu la mosaïque ethnique caractérisant la vallée du Dra, il n'est pas étonnant de constater que I'ADEDRA représente le fief des élites politiques locales arabophones, celles des Chorfas de la zaouïa de Tamgroute pour l'essentiel, au détriment des autres groupes ethniques et tribaux, Aït Atta, Drawa et Aït Sedrate en l'occurrence.

De la vallée du Dra à celle de Ziz, nous sommes face à un prototype d'agents sectoriels qui, convertis en courtiers en développement, n'ignorent pas la sphère conventionnelle de la politique, mais ils ont le sentiment d'avoir choisi en dehors ou contre elle (Pascon, 1980: 5). La dépolitisation est une autre façon de faire et penser la politique. Dans cette perspective, les paroles du président de l'association Addi Ouznou pour le développement sont révélatrices, puisqu'il pose non politiquement des choses qui sont du ressort de la politisation. Par rapport d'emblée à I'INDH, la participation au CLDH, dans le conseil communal, est présumée secondaire. "Elle (l'association) est membre, mais notre participation est formelle " (entretien, le président de l'association Addi Ouzenou, El kheng, le 16 janvier 2010). Mais, envers l'arène politique conventionnelle, les leaders soutiennent un certain apolitisme :

"Notre association a acquis une grande confiance. Au moment de la compagne électorale, nous clorons le siège de l'association. Nous faisons ce que la commune est sommée de faire. [...] Et les associations ont le même avenir que les communes " (entretien, Ibid).

${ }^{21}$ Créé en 1958 par Mahjoubi Aherdan, le mouvement populaire est considéré comme un parti du monde rural, des régions amazighones (Moyen Atlas, Sud-Est et le Rif) et des élites rurales issues de grandes familles de notables et de caïds. Ce parti a connu d'amples scissions. En 2001, le Mouvement national populaire et l'Union démocratique sont fusionnés dans le Mouvement populaire, parti d'origine, et ce, dans le cadre d'un champ partisan multipolaire et fragmenté. 
Dans la pratique du courtage de "retour au peuple " à travers I'interface associative, ce que les techniciens et ingénieurs véhiculent de dépolitisant et de neutralisant va totalement à l'encontre des logiques sociales différenciées et des arènes villageoises sur-politisées (Bako-Arifari et Le Meur, 2002 : 147). A ce sujet, considérons un exemple évocateur. II s'agit de l'association Oasis Ziz pour le développement créée en 2000 à qsar Douira d'Aoufous. Lauréat d'une école de génie civil, MB est technicien dans la commune rurale d'Ertab. Contrairement au cas précédent, cette association dont il est le leader est membre à part entière du Comité local du développement humain. Selon ses dires :

"Elle est l'intermédiaire qui facilite l'activité et les tâches des plans communaux du développement (PDC). Les élus s'accommodent bien avec l'association » (entretien, Aoufous, le 6 janvier 2010).

Dans ces interstices, le technicien devenu courtier associatif et coopératif défend des stratégies de neutralité dans la mise à jour des pratiques du courtage. Mais, paradoxalement, il est tiraillé entre un champ de bataille des savoirs (Battlefields of knowledge) et des rencontres à l'interface (Encounters at the interface) (Long, $2001:$ 70, 107, 243). Un champ où s'entremêlent et se contredisent diverses rationalités : politicienne, lignagère, ethnique, spatiale (celle du douar), technique et développementaliste. Le cas de la coopérative de commercialisation des dattes fondée par le technicien illustre cet enchevêtrement des rationalités. Un tiers d'élus communaux sont membres du bureau. La coopérative porte le nom de Zrigat, douar dont les membres (sept) et les adhérents (60\%) sont majoritaires. Les projets de la coopérative sont implantés dans le douar de Blaghma qui est désormais minoritaire en termes d'adhérents et de membres. L'ensemble de ces rationalités influe sur la composition du conseil communal.

Données ethnographiques à l'appui, nous avons démontré l'hypothèse selon laquelle la neutralisation et son corollaire qui est la dépolitisation ne sont en fin de compte que d'autres manières de faire de la politique et de pratiquer la participation. Ces dernières contribuent à construire la stratégie d'outsider et remodèlent l'espace politique (Abélès, 1986 : 231). Ce sont des points de vue politiques et des positions de pouvoir qui ne se différencient pas de celles de la politisation/notabilisation du courtage. Ultimement, suivant notre analyse, on peut affirmer que derrière les stra- 
tégies de neutralisation se dissimulent les premiers pas dans un processus de notabilisation (Bierschenk et al., 2000 : 32).

Ainsi, les courtiers, qu'ils soient généralistes ou agents sectoriels, qui gomment les frontières du politique et rejettent les formes conventionnelles de la participation politique, sont donc des entrepreneurs de la dépolitisation. Ils s'érigent en porte-parole de l'intérêt général local ou régional, qui se construit sur fond des activités courtières. Le décryptage des logiques représentationnelles révèle que ces dernières constituent différentes façons de concevoir le rejet d'entrée dans l'arène politique instituée (Olivier de Sardan, 1995 : 126), mais aussi les mécanismes de distanciation (neutralité). En effet, ces logiques influent sur la conception même de la relation entre gouvernants et gouvernés et, partant, sur les dispositions prises à l'égard des cadres institués de la politique.

Concernant les généralistes, ils endossent la fonction de porteurs sociaux de projets à travers les interventions sociales du développement. Ils définissent les circonstances de leur redéploiement dans les arènes où ils interviennent et vis-à-vis des groupes au titre et pour le compte desquels ils se dévouent. À propos des agents sectoriels, le " retour au peuple ", qui - de leur point de vue - fait plus figure d'un mal nécessaire que d'un partenaire à part entière (Singleton, $1995: 269$ ), les place face à un dilemme : une injonction paradoxale entre, d'une part, le retrait de la pure technique et du fait transcendantal des projets et, d'autre part, la distance envers des arènes de pouvoirs locaux. Néanmoins, l'examen des données relatives à leurs manières de penser la neutralité montre à quel point ils jouent à la fois le rôle d'insider et d'outsider envers la logique bureaucratique, celle de la productivité/profit et la logique associative et populaire ${ }^{22}$. La reconversion circulaire induit qu'ils se servent des ressources tirées dans la première logique/arène, dans le but d'en remporter d'autres dans la seconde (Bailey, 1971 : 159) et vice versa. S'agit-il là encore de nouveaux notables ? De par la gestion de cet entre-deux, produit de la confrontation entre le dispositif sectoriel de l'Etat et le leadership de la société civile (Chauveau, Le Pape et Olivier de Sardan, 2000: 153), nous pouvons répondre par l'affirmative. Ils sont parmi les nouveaux acteurs locaux prépondérants. Un

22 Pour ces logiques, voir Pascon (1980 : 36). 
parti pris que Paul Pascon sous-estime lorsqu'il affirme que les techniciens ou ingénieurs aideraient à faire naître et à faire prospérer des prépondérants locaux qui font une politique de notables n'ayant rien à voir avec les projets techniques (Pascon, 1980: 6). Désormais, ces agents sectoriels, qui " reviennent au peuple " via le secteur associatif, incarnent de nouveaux notables - de nouveaux acteurs locaux prépondérants -, en ce qu'ils sont :

"[...] dotés d'un ressort territorial, d'un groupe d'interconnaissance, en s'assurant un capital social de relations utiles et un capital symbolique de reconnaissance grâce à la forme particulière d'échange... comme un service, à un usager en plus ou tel de ses protégés (populations-cibles). » (Bourdieu, 2000 : 162)

\section{Conclusion}

La reconversion des ressources ou capitaux tirés du courtage en développement conforte la thèse de la fabrique et de la consolidation des élites dans les arènes locales du Sud-Est marocain. Généralement, quelle que soit la stratégie de tel ou tel courtier, l'imbrication des logiques et des positions sociales va de pair avec des formes différenciées de drainage et d'accumulation, ainsi que des "façons de concevoir " la reconversion, la qualification et la mise en valeur des ressources de divers degrés.

Le franchissement des frontières entre courtage et notabilité illustre les connexions qui émergent entre la "politique des problèmes" - et aussi " celle des besoins » - d'un côté, et la compétition électorale de l'autre, ou entre le "marché » du développement et le "marché » politique. A la lumière des cas empiriques cités, ce franchissement encastre (e.i. au sens embedded) la carrière du courtier dans un parcours politique en plein lancement. Dans un contexte de surpolitisation des arènes, les courtiers en développement accèdent de fait aux cercles de la notabilité. Ils endossent la fonction du courtage dans l'implémentation des projets de développement et de l'action publique locale en vue de remporter des trophées politiques, y compris les élections communales et législatives. Ils ne gagnent pas celles-ci pour endosser celle-là. Le courtage engendre là aussi deux logiques élitaires : celle d'évincement et de remplacement d'une part, et celle d'alliance et de cohabitation d'autre part. La première considère les courtiers comme étant une élite de substitution. Ici, les activités 
courtières émancipent de nouveaux venus au sein de ces arènes, puisqu'elles les dotent de capacités techniques pour infiltrer les rangs de l'élite. De nouvelles élites rurales émergent et bousculent le monopole d'anciens notables établis. Dans la deuxième, ces derniers " font " affaire avec ceux qu'ils considèrent comme de nouveaux rivaux. Ces courtiers attendent d'eux une promotion afin de prendre position au sein de la logique prééminente (ascription) de la hiérarchie traditionnelle. Les notables s'aperçoivent en contrepartie qu'il est de l'intérêt de la jema'a (assemblée délibérative traditionnelle), dont ils sont de facto des leaders, de s'adjoindre une élite qui est, de leur point de vue, efficace, instruite, compétente, performante et docile.

Même dans les cas contrastés où les contours du politique s'effacent, les positions du pouvoir se dissimulent. Cet effacement ou gommage ne signifie-t-il pas la reconversion manquée des ressources générées du courtage ? À la lumière de ce qui précède, il faut distinguer deux voies. La première est celle des courtiers qui entretiennent un sens caché ou un intérêt déguisé à l'égard de l'arène politique instituée. II s'agit d'une catégorie de courtiers pour qui cette arène ne représente pas un enjeu stratégique, pardelà les savoir-faire experts dont ils se prévalent. Pour eux, il existe $\mathrm{d}^{\prime}$ autres univers/arènes où ils peuvent circuler, recycler et reconvertir des ressources disponibles, étant donné qu'ils changent de casquette (institutionnelle, administrative, technicienne, associative, coopérative et partisane) selon l'occasion. Et l'occasion - le projet de développement - fait le larron - le courtier, nous révèle Le Meur (2008:6). C'est pour cette raison qu'à l'égard des pouvoirs publics, des élites politiques en place, des acteurs institutionnels et des autres courtiers, la stratégie d'outsider plaît à ces courtiers. La seconde voie renvoie aux courtiers qui possèdent des capacités techniques mais qui sont dépourvus d'ancrage local, de réseaux familiaux, tribaux ou ethniques et partisans. Leurs stratégies de neutralité ou de dépolitisation dans les pratiques du courtage en développement cachent à cet égard des reconversions manquées et des instrumentalisations réciproques échouées entre "la politique des problèmes et des besoins » et « la politique électorale». 


\section{Bibliographie}

ABELES M., 1986, "Le degré zéro de la politique ", Études rurales, 101-102: 231269.

ADEDRA, 2005, "Activités réalisées, leçons retenues et perspectives d'avenir ", Rapport de synthèse (1996-2004).

AïT-AOUDIA M., BENNANI-CHRAÏBI M., CONTAMIN J.-G., 2010, « Contribution à une histoire sociale de la conception lagroyenne de la politisation ", Critique internationale, 3/48: 207-220.

ALIFRIQUI M., 2012, "Projet Conservation de la Biodiversité dans le Saghro ", Rapport d'évaluation finale du projet, (MOR/SGP/OP4/Y2/RAF/2008/03 / MOR/SGP/OP4/Y2/CORE/2008/02), PMF/FEM-PNUD/ Fédération Bougafer, FEF et SGP, $115 \mathrm{p}$.

AL-SAYYID SAID M., 2004, "Culture de la relation entre le civil et le politique dans les ONG », in BEN NEFISSA S., ABD AL-FATTAH N., HANAFI S., MILANI C. (éds.), ONG et gouvernance dans le monde arabe, Paris, Karthala-CEDEJ : 65-79.

ARNAUD L. et GUINNET C., 2005, "Introduction: Les frontières du politique. Enquêtes sur les processus de politisation et de dépolitisation ", in ARNAUD L. et GUINNET C. (éds.), Les frontières du politique. Enquêtes sur les processus de politisation et de dépolitisation, Rennes, Presses universitaires de Rennes : 11-25.

BAILEY F.G., 1971, Les règles du jeu politique. Etude anthropologique, Paris, PUF.

BAKO-ARIFARI N. et LE MEUR P.-Y., 2002, "Une anthropologie sociale des dispositifs du développement ", in BARE J.-F. (éd.), L'évaluation des politiques de développement. Approches pluridisciplinaires, Paris, Editions L'Harmattan : 121-173.

BALME R., 1987, " La participation aux associations et le pouvoir municipal ", Revue française de sociologie, 28/4 : 601-639.

BENIDIR M., 2010, "Leaders associatifs et élus locaux au Maroc : épreuves de face à face et controverses dans les arènes du développement ", in CATUSSE M. et VAIREL F. (éds.), "Le Maroc de Mohamed VI. Mobilisations et action publique », Politique africaine, dossier, $120: 87-104$.

BENIDIR M., 2011, "Entre notabilité et courtage. Les dynamiques associatives face au développement local dans le versant sud du Haut-Atlas (Ouarzazate) ", Revue marocaine de science politique, 2 : 69-101. 
BENIDIR M., 2012, "Courtage collectif en développement et réseaux associatifs dans la région Drâa-Tafilalet ", in AIT HAMZA M. (éd.), "Culture et développement. Ressources, savoirs et savoir-faire locaux », Asinag, Revue de l'IRCAM, dossier, 7 : 31-56.

BENIDIR M., 2013, « Députés tribaux et/ou ethniques? La mobilisation de l'élite parlementaire dans l'arène électorale d'Ouarzazate ", in Madani M. (éd.), "Les élections et les modes du scrutin au Maroc ", Revue marocaine des sciences politiques et sociales, dossier, 5-6 : 333-374.

BENIDIR M., 2014, "Biodiversité et biopolitique. Conflit pastoral autour d'un nouvel agdal sur le versant sud du Haut Atlas (Maroc) ", in BREDA C., CHAPLIER M., HERMESSE J. et PICCOLI E. (éds.), Terres (dés)humanisées : ressources et climat, Coll. Investigations d'Anthropologie Prospective, 10, Louvain-la-Neuve, Academia-L'Harmattan : 343-368.

BENNANI-CHRAÏBI M., 2003, "Parcours, cercles et médiations à Casablanca. Tous les chemins mènent à l'action associative de quartier ", in BENNANICHRAÏBI M. et FILLIEULE O. (éds.), Résistances et protestations dans les sociétés musulmanes, Paris, Presses de Sciences Po. : 293-352.

BENNANI-CHRAÏBI M., 2004, "Représenter et mobiliser aux élections au Maroc » in BENNANI-CHRAÏBI M., CATUSSE M., SANTUCCI J.-C. (éds.), Scènes et coulisses de l'élection au Maroc: les législatives de 2002, introduction, Paris/Aix-en-Provence, Editions Karthala/IREMAM : 15-53.

BENNANI-CHRAÏBI M., 2011, " Jeux de miroir de la "politisation": les acteurs associatifs de quartier à Casablanca ", Critique internationale, 50/1 : 5571.

BERDOUZI M., 1987, « Problèmes d'interprétation et fondements conceptuels des approches anglo-américaines concernant le Maroc. Etude d'un corpus de travaux d'anthropologie et de sciences politiques ", tome I et II, thèse de doctorat d'Etat en Droit public (sciences politiques), Université Mohamed V, FSJES, Rabat, 624 pp.

BERRIANE Y., 2009, «Intermédiations stratégiques. L'engagement des militantes associatives locales dans la campagne pour les législatives marocaines de 2007 ", in ZAKI L. (éd.), Terrains de campagne au Maroc. Les législatives de 2007, Tunis-Paris, IRMC-Karthala : 161-191.

BIERSCHENK T., CHAUVEAU J.-P. et OLIVIER DE SARDAN J.-P., 2000, « Les courtiers entre développement et État » in BIERSCHENK T., CHAUVEAU J.-P. et OLIVIER DE SARDAN J.-P. (éds.), Courtiers en développement. Les villages africains en quête de projets, Paris, Apad/Karthala : 5-42.

BLUNDO G., 1995, "Les courtiers locaux du développement en milieu rural sénégalais ", Cahiers d'études africaines, 137, XXXV-1 : 73-99. 
BLUNDO G., 1998, «Logiques de gestion publique dans la décentralisation sénégalaise : participation factionnelle et ubiquité réticulaire », Bulletin de l'APAD, 15 : 21-47.

BLUNDO G., 2000, « Elus locaux et courtiers en développement au Sénégal. Trajectoires politiques, modes de légitimation et stratégies d'alliance ", in BIERSCHENK T., CHAUVEAU J.-P. et OLIVIER DE SARDAN J.-P. (éds.), Les courtiers en développement. Les villages africains en quête de projets, Paris, APAD-Karthala : 71-100.

BONO I., 2009, "Pauvreté, exception, participation. Mobilisation et démobilisations "dans le cadre de I'INDH" au Maroc ", in CATUSSE M., DESTREMAU B. et VERDIER E. (éds.), L'Etat face aux débordements du social au Maghreb. Formation, travail et protection sociale, Paris/Aix-enProvence, IREMAM/Karthala : 229-250.

BOUCHER Y. et VILLALBA B., 1990, "Le militant, la compétence et l'éthique : les conditions de l'investiture chez les Verts ", Politix, 9/3 : 37-43.

BOURDARIAS F., 2003, "ONG et développement des élites", Journal des anthropologues, 94-95:23-52.

BOURDIEU P., 1994, "Stratégies de reproduction et modes de domination ", Actes de recherches en sciences sociales, 105 : 3-12.

BOURDIEU P., 2000, "Le champ des pouvoirs locaux ", in BOURDIEU P., Les structures sociales de l'économie, Paris, Coll. Liber, Éditions du Seuil : 155180.

BOURQIA R., 2011, Culture politique au Maroc. À l'épreuve des mutations, Paris, Coll. Logiques politiques, L'Harmattan.

CATUSSE M., 2002, "Le charme discret de la société civile. Ressorts politiques de la formation d'un groupe dans le Maroc "ajusté" ", Revue internationale de politique comparée, 2 : 297-318.

CATUSSE M. et KARAM K., 2009, « Le développement contre la représentation ? La technicisation du gouvernement local au Liban et au Maroc ", in CAMAU M. et MASSARDIER G. (éds.), Démocraties et autoritarismes. Fragmentation et hybridation des régimes, Paris, Karthala/CSPC : 85-120.

CATUSSE M. et ZAKI L., 2009, " Gestion communale et clientélisme moral au Maroc: les politiques du Parti de la justice et du développement ", Critique internationale, 42/1 : 73-91.

CHAUVEAU J.-P., LE PAPE M. et OLIVIER DE SARDAN J.-P., 2000, « La pluralité des normes et leurs dynamiques en Afrique. Implications pour les politiques publiques », in WINTER G. (éd.), Inégalités et politiques publiques en 
Afrique : pluralité des normes et jeux d'acteurs, Paris, IRD-Karthala : 145162.

CHEYNIS E., 2008, " L'espace des transformations de l'action associative au Maroc. Réforme de l'action publique, investissements militants et légitimité internationale ", thèse de doctorat de sciences sociales, Université Paris I Panthéon-Sorbonne.

DAHOU T., 2003, "Clientélisme et ONG. Un cas sénégalais ", in "Les ONG, médiations politiques et globalisation ", Journal des anthropologues, dossier, 94-95: 145-163.

DE MAILLARD J., 2006, "La conduite des politiques publiques à l'épreuve des temporalités électorales. Quelques hypothèses exploratoires ", Pôle Sud, $25: 39-53$.

FAURE A., 1997, « Les apprentissages du métier d'élu local: la tribu, le système et les arènes ", Pôle Sud, 7 : 72-79.

GAXIE D., 1978, Le sens caché. Inégalités culturelles et ségrégations politiques, Paris, Éditions du Seuil.

GELLNER E., 1986, "L'animal qui évite les gaffes ou un faisceau d'hypothèses ", in BIRBAUM P. et LECA J. (éds.), Sur l'individualisme, Paris, Presses de la Fondation des sciences politiques : 27-44.

GESCHIERE P., 1996, "Sorcellerie et politique: les pièges du rapport élitevillages ", Politique africaine, $63: 82-96$.

ION J., 1997, La fin des militants, Paris, Les Editions de l'Atelier/Editions ouvrières.

LAGROYE J., 2003, "Les processus de politisation ", in LAGROYE J. (éd.), La politisation, Paris, Belin : 359-372.

LAVIGNE DELVILLE P., 2000, "Courtiers en développement ou entrepreneurs politiques ? Les responsables d'associations villageoises de développement dans la région d'émigration internationale (Sénégal, Mali) ", in BIERSCHENK T., CHAUVEAU J.-P. et OLIVIER DE SARDAN J.-P. (éds.), Courtiers en développement. Les villages africains en quête de projets, Paris, Apad/Karthala : 165-187.

LE MEUR P.Y., 2008, "Le développement comme constructivisme. Point de vue anthropologique ", Sociétés politiques comparées, 8, 41 p.

LONG N., 2001, Development Sociology: Actor Perspectives, London and New York: Routlege.

MENDRAS H., 1962, "Politisation, dépolitisation et repolitisation en milieu rural ", in VEDEL G. (éd.), La dépolitisation. Mythe ou réalité ?, Cahiers de la FNSP, 120, Paris, Armand Colin : 251-265. 
MERLE M., 1962, "Inventaire des apolitismes en France » in VEDEL G. (éd.), La dépolitisation. Mythe ou réalité ?, Cahiers de la FNSP, 120, Paris, Armand Colin : 43-60.

NAY O. et SMITH A., 2002, "Les intermédiaires en politique. Médiation et jeux d'institutions ", in NAY O. et SMITH A. (éds.), Le gouvernement $d u$ compromis. Courtiers et généralistes de l'action politique, Paris, Economica : 1-21.

OLIVIER DE SARDAN J.-P., 1995, Anthropologie et développement. Essai en socioanthropologie du changement social, Paris, APAD-Karthala.

OLIVIER DE SARDAN J.-P., 2008, La rigueur du qualificatif. Les contraintes empiriques de l'interprétation socio-anthropologique, Louvain-La-Neuve, Academia-Bruylant, Coll. Anthropologie Prospective, 3.

PASCON P., 1980, Études rurales. Idées et enquêtes sur la campagne marocaine, Rabat, SMER.

SINGLETON M., 1995, " Un anthropologue et des agronomes au Maroc. Journal de voyage ", Alliages, 24-25: 264-271.

VEDEL G., 1962, "Rapport introductif », in VEDEL G. (dir.), La dépolitisation. Mythe ou réalité ?,Cahiers de la FNSP, 120, Paris, Armand Colin : 5-24.

ZAINABI A. T., 2003, "La vallée du Dra: Développement alternatif et action communautaire ", World Bank Report. Dynamic De. velopment in a Sustainable World, Background Paper

ZAINABI A.T. et OUHAJOU L., 2005, "Portée et limite d'une participation citoyenne: les ONG de la vallée de Dra Moyen/Sud-Est marocain ", in HAMMAM M. et al. (éds.), L'environnement au Maroc. Données historiques et perspectives de développement. Le cas de la région du Dra, IRCAM-CEHE, Série Colloque et Séminaires, 9, Rabat : 7-21.

\section{Aiviviv}

\section{Mohammed Benidir est doctorant}

Faculté des sciences juridiques, économiques et sociales, Université Mohammed V Rabat-Agdal Institut des sciences humaines et sociales, Université de Liège

E-mail : M.Benidir@doct.ulg.ac.be 